\title{
Multi-curve Modelling Using Trees
}

\author{
John Hull and Alan White
}

\begin{abstract}
Since 2008 the valuation of derivatives has evolved so that OIS discounting rather than LIBOR discounting is used. Payoffs from interest rate derivatives usually depend on LIBOR. This means that the valuation of interest rate derivatives depends on the evolution of two different term structures. The spread between OIS and LIBOR rates is often assumed to be constant or deterministic. This paper explores how this assumption can be relaxed. It shows how well-established methods used to represent one-factor interest rate models in the form of a binomial or trinomial tree can be extended so that the OIS rate and a LIBOR rate are jointly modelled in a threedimensional tree. The procedures are illustrated with the valuation of spread options and Bermudan swap options. The tree is constructed so that LIBOR swap rates are matched.
\end{abstract}

Keywords OIS $\cdot$ LIBOR $\cdot$ Interest rate trees $\cdot$ Multi-curve modelling

\section{Introduction}

Before the 2008 credit crisis, the spread between a LIBOR rate and the corresponding OIS (overnight indexed swap) rate was typically around 10 basis points. During the crisis this spread rose dramatically. This led practitioners to review their derivatives valuation procedures. A result of this review was a switch from LIBOR discounting to OIS discounting.

Finance theory argues that derivatives can be correctly valued by estimating expected cash flows in a risk-neutral world and discounting them at the risk-free rate. The OIS rate is a better proxy for the risk-free rate than LIBOR. ${ }^{1}$ Another argument

\footnotetext{
${ }^{1}$ See for example Hull and White [15].

J. Hull (殴 · A. White

Joseph L. Rotman School of Management, University of Toronto, Toronto, ON, Canada

e-mail: hull@ rotman.utoronto.ca
}

\author{
A. White \\ e-mail: awhite@rotman.utoronto.ca \\ (C) The Author(s) 2016 \\ K. Glau et al. (eds.), Innovations in Derivatives Markets, Springer Proceedings \\ in Mathematics \& Statistics 165, DOI 10.1007/978-3-319-33446-2_9
}


(appealing to many practitioners) in favor of using the OIS rate for discounting is that the interest paid on cash collateral is usually the overnight interbank rate and OIS rates are longer term rates derived from these overnight rates. The use of OIS rates therefore reflects funding costs.

Many interest rate derivatives provide payoffs dependent on LIBOR. When LIBOR discounting was used, only one rate needed to be modelled to value these derivatives. Now that OIS discounting is used, more than one rate has to be considered. The spread between OIS and LIBOR rates is often assumed to be constant or deterministic. This paper provides a way of relaxing this assumption. It describes a way in which LIBOR with a particular tenor and OIS can be modelled using a three-dimensional tree. ${ }^{2}$ It is an extension of ideas in the many papers that have been written on how one-factor interest rate models can be represented in the form of a two-dimensional tree. These papers include Ho and Lee [9], Black, Derman, and Toy [3], Black and Karasinski [4], Kalotay, Williams, and Fabozzi [18], Hainaut and MacGilchrist [8], and Hull and White [11, 13, 14, 16].

The balance of the paper is organized as follows. We first describe how LIBOROIS spreads have evolved through time. Second, we describe how a three-dimensional tree can be constructed to model both OIS rates and the LIBOR-OIS spread with a particular tenor. We then illustrate the tree-building process using a simple threestep tree. We investigate the convergence of the three-dimensional tree by using it to calculate the value of options on the LIBOR-OIS spread. We then value Bermudan swap options showing that in a low-interest-rate environment, the assumption that the spread is stochastic rather than deterministic can have a non-trivial effect on valuations.

\section{The LIBOR-OIS Spread}

LIBOR quotes for maturities of one-, three-, six-, and 12-months in a variety of currencies are produced every day by the British Bankers' Association based on submissions from a panel of contributing banks. These are estimates of the unsecured rates at which AA-rated banks can borrow from other banks. The $T$-month OIS rate is the fixed rate paid on a $T$-month overnight interest rate swap. In such a swap the payment at the end of $T$-months is the difference between the fixed rate and a rate which is the geometric mean of daily overnight rates. The calculation of the payment on the floating side is designed to replicate the aggregate interest that would be earned from rolling over a sequence of daily loans at the overnight rate. (In U.S. dollars, the overnight rate used is the effective federal funds rate.) The LIBOR-OIS spread is the LIBOR rate less the corresponding OIS rate.

\footnotetext{
${ }^{2}$ At the end of Hull and White [17] we described an attempt to do this using a two-dimensional tree. The current procedure is better. Our earlier procedure only provides an approximate answer because the correlation between spreads at adjacent tree nodes is not fully modelled.
} 
LIBOR-OIS spreads were markedly different during the pre-crisis (December 2001-July 2007) and post-crisis (July 2009-April 2015) periods. This is illustrated in Fig. 1. In the pre-crisis period, the spread term structure was quite flat with the 12month spread only about 4 basis points higher than the one-month spread on average. As shown in Fig. 1a, the 12-month spread was sometimes higher and sometimes lower than one-month spread. The average one-month spread was about 10 basis points during this period. Because the term structure of spreads was on average fairly flat and quite small, it was plausible for practitioners to assume the existence of a single LIBOR zero curve and use it as a proxy for the risk-free zero curve. During the postcrisis period there has been a marked term structure of spreads. As shown in Fig. 1b, it is almost always the case that the spread curve is upward sloping. The average one-month spread continues to be about 10 basis points, but the average 12-month spread is about 62 basis points.

There are two factors that explain the difference between LIBOR rates and OIS rates. The first of these may be institutional. If a regression model is used to extrapolate the spread curve for shorter maturities, we find the one-day spread in the post-crisis period is estimated to be about 5 basis points. This is consistent with the spread between one-day LIBOR and the effective fed funds rate. Since these are both rates that a bank would pay to borrow money for $24 \mathrm{~h}$, they should be the same. The 5 basis point difference must be related to institutional practices that affect the two different markets. ${ }^{3}$

Given that institutional differences account for about 5 basis points of spread, the balance of the spread must be attributable to credit. OIS rates are based on a continually refreshed one-day rate whereas $\tau$-maturity LIBOR is a continually refreshed $\tau$-maturity rate. ${ }^{4}$ The difference between $\tau$-maturity LIBOR and $\tau$-maturity OIS then reflects the degree to which the credit quality of the LIBOR borrower is expected to decline over $\tau$ years. ${ }^{5}$ In the pre-crisis period the expected decline in the borrower credit quality implied by the spreads was small but during the post-crisis period it has been much larger.

The average hazard rate over the life of a LIBOR loan with maturity $\tau$ is approximately

$$
\bar{\lambda}=\frac{L(\tau)}{1-R}
$$

where $L(\tau)$ is the spread of LIBOR over the risk-free rate and $R$ is the recovery rate in the event of default. Let $h$ be the hazard rate for overnight loans to high quality financial institutions (those that can borrow at the effective fed funds rate). This will also be the average hazard rate associated with OIS rates.

\footnotetext{
${ }^{3}$ For a more detailed discussion of these issues see Hull and White [15].

${ }^{4} \mathrm{~A}$ continually refreshed $\tau$-maturity rate is the rate realized when a loan is made to a party with a certain specified credit rating (usually assumed in this context to be AA) for time $\tau$. At the end of the period a new $\tau$-maturity loan is made to a possibly different party with the same specified credit rating. See Collin-Dufresne and Solnik [6].

${ }^{5}$ It is well established that for high quality borrowers the expected credit quality declines with the passage of time.
} 
(a)

30

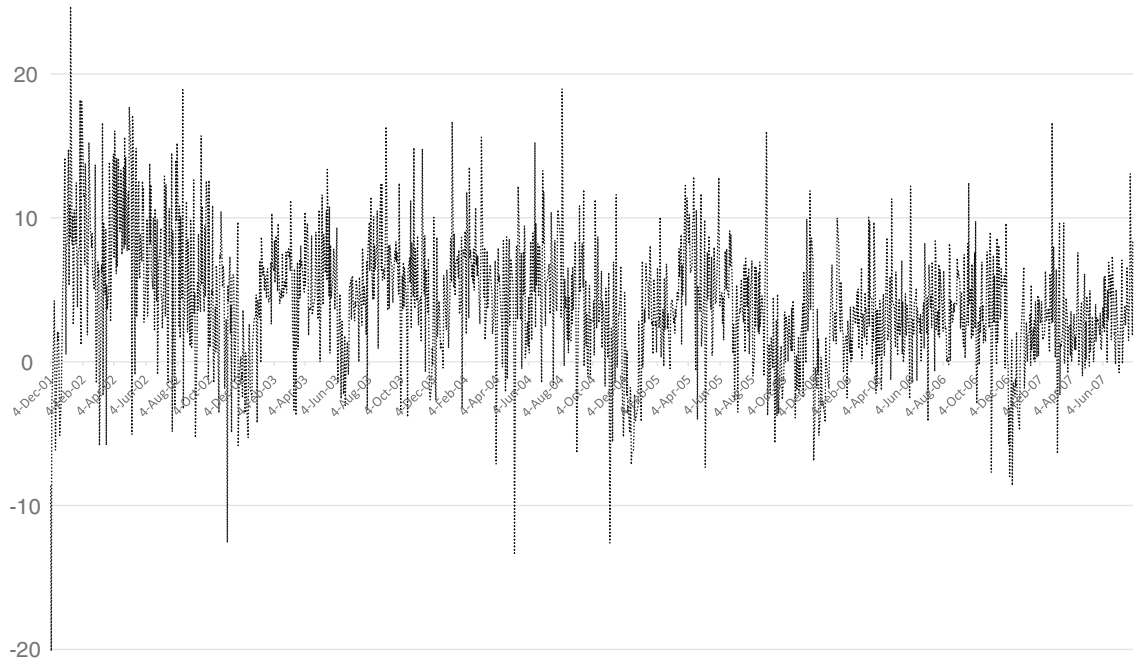

(b)

14

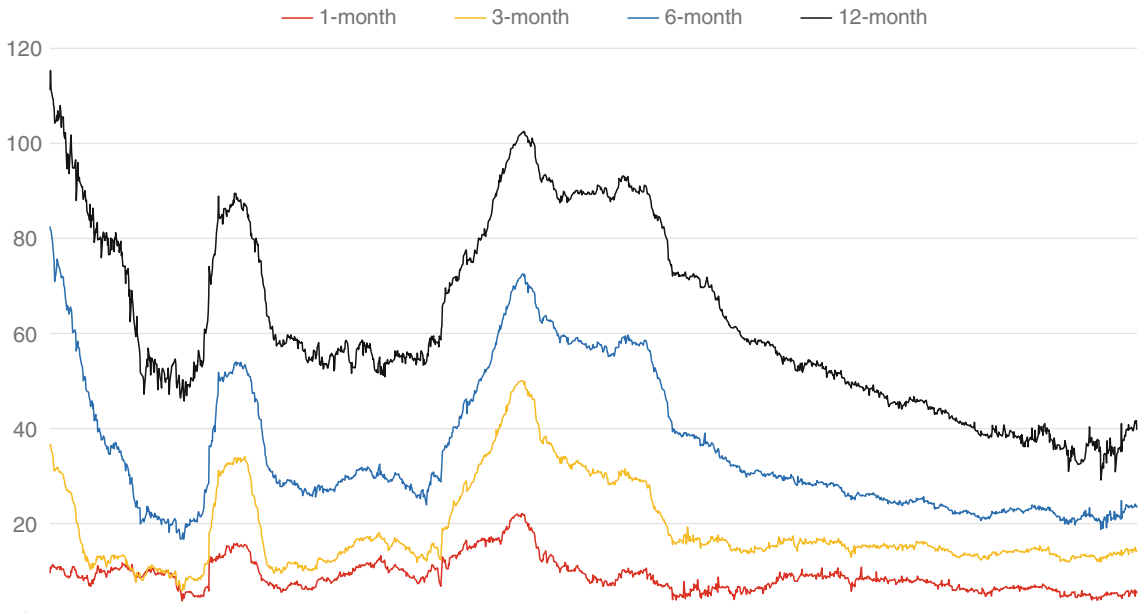
0

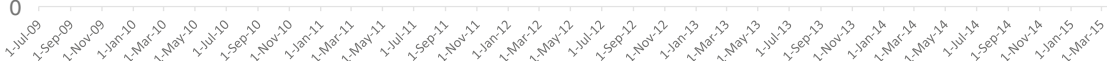

Fig. 1 a Excess of 12-month LIBOR-OIS spread over one-month LIBOR-OIS spread December 4, 2001-July 31, 2007 period (basis points). Data Source: Bloomberg. b Post-crisis LIBOR-OIS spread for different tenors (basis points). Data Source: Bloomberg 
Define $L^{*}(\tau)$ as the spread of LIBOR over OIS for a maturity of $\tau$ and $O(\tau)$ as the spread of OIS over the risk-free rate for this maturity. Because $L(\tau)=L^{*}(\tau)+O(\tau)$

$$
\bar{\lambda}=\frac{L^{*}(\tau)+O(\tau)}{1-R}=h+\frac{L^{*}(\tau)}{1-R}
$$

This shows that when we model OIS and LIBOR we are effectively modelling OIS and the difference between the LIBOR hazard rate and the OIS hazard rate.

One of the results of the post-crisis spread term structure is that a single LIBOR zero curve no longer exists. LIBOR zero curves can be constructed from swap rates, but there is a different LIBOR zero curve for each tenor. This paper shows how OIS rates and a LIBOR rate with a particular tenor can be modelled jointly using a three-dimensional tree. ${ }^{6}$

\section{The Methodology}

Suppose that we are interested in modelling OIS rates and the LIBOR rate with tenor of $\tau$. (Values of $\tau$ commonly used are one month, three months, six months and 12 months.) Define $r$ as the instantaneous OIS rate. We assume that some function of $r, x(r)$, follows the process

$$
\mathrm{d} x=\left[\theta(t)-a_{r} x\right] \mathrm{d} t+\sigma_{r} \mathrm{~d} z_{r}
$$

This is an Ornstein-Uhlenbeck process with a time-dependent reversion level. The function $\theta(t)$ is chosen to match the initial term structure of OIS rates; $a_{r}(\geq 0)$ is the reversion rate of $x ; \sigma_{r}(>0)$ is the volatility of $r$; and $\mathrm{d} z_{r}$ is a Wiener process. ${ }^{7}$

Define $s$ as the spread between the LIBOR rate with tenor $\tau$ and the OIS rate with tenor $\tau$ (both rates being measured with a compounding frequency corresponding to the tenor). We assume that some function of $s, y(s)$, follows the process:

$$
\mathrm{d} y=\left[\phi(t)-a_{s} y\right] \mathrm{d} t+\sigma_{s} \mathrm{~d} z_{s}
$$

This is also an Ornstein-Uhlenbeck process with a time-dependent reversion level. The function $\phi(t)$ is chosen to ensure that all LIBOR FRAs and swaps that can be entered into today have a value of zero; $a_{s}(\geq 0)$ is the reversion rate of $y ; \sigma_{s}(>0)$ is

\footnotetext{
${ }^{6}$ Extending the approach so that more than one LIBOR rate is modelled is not likely to be feasible as it would involve using backward induction in conjunction with a four (or more)-dimensional tree. In practice, multiple LIBOR rates are most likely to be needed for portfolios when credit and other valuation adjustments are calculated. Monte Carlo simulation is usually used in these situations.

${ }^{7}$ This model does not allow interest rates to become negative. Negative interest have been observed in some currencies (particularly the euro and Swiss franc). If $-e$ is the assumed minimum interest rate, this model can be adjusted so that $x=\ln (r+e)$. The choice of $e$ is somewhat arbitrary, but changes the assumptions made about the behavior of interest rates in a non-trivial way.
} 
the volatility of $s$; and $\mathrm{d} z_{s}$ is a Wiener process. The correlation between $\mathrm{d} z_{r}$ and $\mathrm{d} z_{s}$ will be denoted by $\rho$.

We will use a three-dimensional tree to model $x$ and $y$. A tree is a discrete time, discrete space approximation of a continuous stochastic process for a variable. The tree is constructed so that the mean and standard deviation of the variable is matched over each time step. Results in Ames [1] show that in the limit the tree converges to the continuous time process. At each node of the tree, $r$ and $s$ can be calculated using the inverse of the functions $x$ and $y$.

We will first outline a step-by-step approach to constructing the three-dimensional tree and then provide more details in the context of a numerical example in Sect. $4 .^{8}$ The steps in the construction of the tree are as follows:

1. Model the instantaneous OIS rate using a tree. We assume that the process for $r$ is defined by Eq. (1) and that a trinomial tree is constructed as described in Hull and White [11, 13] or Hull [10]. However, the method we describe can be used in conjunction with other binomial and trinomial tree-building procedures such as those in Ho and Lee [9], Black, Derman and Toy [3], Black and Karasinski [4], Kalotay, Williams and Fabozzi [18] and Hull and White [14, 16]. Tree building procedures are also discussed in a number of texts. ${ }^{9}$ If the tree has steps of length $\Delta t$, the interest rate at each node of the tree is an OIS rate with maturity $\Delta t$. We assume the tree can be constructed so that both the LIBOR tenor, $\tau$, and all potential payment times for the instrument being valued are multiples of $\Delta t$. If this is not possible, a tree with varying time steps can be constructed. ${ }^{10}$

2. Use backward induction to calculate at each node of the tree the price of an OIS zero-coupon bond with a life of $\tau$. For a node at time $t$ this involves valuing a bond that has a value of $\$ 1$ at time $t+\tau$. The value of the bond at nodes earlier than $t+\tau$ is found by discounting through the tree. For each node at time $t+\tau-\Delta t$ the price of the bond is $e^{-r \Delta t}$ where $r$ is the ( $\Delta t$-maturity) OIS rate at the node. For each node at time $t+\tau-2 \Delta t$ the price is $e^{-r \Delta t}$ times a probability-weighted average of prices at the nodes at time $t+\tau-\Delta t$ which can be reached from that node, and so on. The calculations are illustrated in the next section. Based on the bond price calculated in this way, $P$, the $\tau$-maturity OIS rate, expressed with a compounding period of $\tau$, is ${ }^{11}$

$$
\frac{1 / P-1}{\tau}
$$

3. Construct a trinomial tree for the process for the spread function, $y$, in Eq. (2) when the function $\phi(t)$ is set equal to zero and the initial value of $y$ is set equal to

\footnotetext{
${ }^{8}$ Readers who have worked with interest rate trees will be able to follow our step-by-step approach. Other readers may prefer to follow the numerical example.

${ }^{9}$ See for example Brigo and Mercurio [5] or Hull [10].

${ }^{10}$ See for example Hull and White [14].

${ }^{11}$ The $r$-tree shows the evolution of the $\Delta t$-maturity OIS rate. Since we are interested in modelling the $\tau$-maturity LIBOR-OIS spread, it is necessary to determine the evolution of the $\tau$-maturity OIS rate.
} 
zero. ${ }^{12}$ We will refer to this as the "preliminary tree". When interest rate trees are built, the expected value of the short rate at each time step is chosen so that the initial term structure is matched. The adjustment to the expected rate at time $t$ is achieved by adding some constant, $\alpha_{t}$, to the value of $x$ at each node at that step. ${ }^{13}$ The expected value of the spread at each step of the spread tree that is eventually constructed will similarly be chosen to match forward LIBOR rates. The current preliminary tree is a first step toward the construction of the final spread tree.

4. Create a three-dimensional tree from the OIS tree and the preliminary spread tree assuming zero correlation between the OIS rate and the spread. The probabilities on the branches of this three-dimensional tree are the product of the probabilities on the corresponding branches of the underlying two-dimensional trees.

5. Build in correlation between the OIS rate and the spread by adjusting the probabilities on the branches of the three-dimensional tree. The way of doing this is described in Hull and White [12] and will be explained in more detail later in this paper.

6. Using an iterative procedure, adjust the expected spread at each of the times considered by the tree. For the nodes at time $t$, we consider a receive-fixed forward rate agreement (FRA) applicable to the period between $t$ and $t+\tau{ }^{14}$ The fixed rate, $F$, equals the forward rate at time zero. The value of the FRA at a node, where the $\tau$-maturity OIS rate is $w$ and the $\tau$-maturity LIBOR-OIS spread is $s$, is ${ }^{15}$

$$
\frac{F-(w+s)}{1+w \tau}
$$

The value of the FRA is calculated for all nodes at time $t$ and the values are discounted back through the three-dimensional tree to find the present value. ${ }^{16}$ As discussed in step 3, the expected spread (i.e., the amount by which nodes are shifted from their positions in the preliminary tree) is chosen so that this present value is zero.

\footnotetext{
${ }^{12} \mathrm{As}$ in the case of the tree for the interest rate function, $x$, the method can be generalized to accommodate a variety of two-dimensional and three-dimensional tree-building procedures.

${ }^{13}$ This is equivalent to determining the time varying drift parameter, $\theta(t)$, that is consistent with the current term structure.

${ }^{14} \mathrm{~A}$ forward rate agreement (FRA) is one leg of a fixed for floating interest rate swap. Typically, the forward rates underlying some FRAs can be observed in the market. Others can be bootstrapped from the fixed rates exchanged in interest rate swaps.

${ }^{15} F, w$, and $s$ are expressed with a compounding period of $\tau$.

${ }^{16}$ Calculations are simplified by calculating Arrow-Debreu prices, first at all nodes of the twodimensional OIS tree and then at all nodes of the three-dimensional tree. The latter can be calculated at the end of the fifth step as they do not depend on spread values. This is explained in more detail and illustrated numerically in Sect. 4.
} 


\section{A Simple Three-Step Example}

We now present a simple example to illustrate the implementation of our procedure. We assume that the LIBOR maturity of interest is 12 months $(\tau=1)$. We assume that $x=\ln (r)$ with $x$ following the process in Eq. (1). Similarly we assume that $y=\ln (s)$ with $y$ following the process in Eq. (2). We assume that the initial OIS zero rates and 12 month LIBOR forward rates are those shown in Table 1. We will build a 1.5 -year tree where the time step, $\Delta t$, equals 0.5 years. We assume that the reversion rate and volatility parameters are as shown in Table 2.

As explained in Hull and White $[11,13]$ we first build a tree for $x$ assuming that $\theta(t)=0$. We set the spacing of the $x$ nodes, $\Delta x$, equal to $\sigma_{r} \sqrt{3 \Delta t}=0.3062$. Define node $(i, j)$ as the node at time $i \Delta t$ for which $x=j \Delta x$. (The middle node at each time has $j=0$.) The normal branching process in the tree is from $(i, j)$ to one of $(i+1, j+1),(i+1, j)$, and $(i+1, j-1)$. The transition probabilities to these three nodes are $p_{u}, p_{m}$, and $p_{d}$ and are chosen to match the mean and standard deviation

Table 1 Percentage interest rates for the examples

\begin{tabular}{l|l|l|l|l}
\hline $\begin{array}{l}\text { Maturity } \\
\text { years })\end{array}$ & OIS zero rate & $\begin{array}{l}\text { Forward } \\
\text { 12-month } \\
\text { LIBOR rate }\end{array}$ & $\begin{array}{l}\text { Forward } \\
\text { 12-month OIS } \\
\text { rate }\end{array}$ & $\begin{array}{l}\text { Forward Spread: } \\
\text { 12-month LIBOR less } \\
12 \text {-month OIS }\end{array}$ \\
\hline 0 & 3.000 & 3.300 & 3.149 & 0.151 \\
\hline 0.5 & 3.050 & 3.410 & 3.252 & 0.158 \\
\hline 1.0 & 3.100 & 3.520 & 3.355 & 0.165 \\
\hline 1.5 & 3.150 & 3.630 & 3.458 & 0.172 \\
\hline 2.0 & 3.200 & 3.740 & 3.562 & 0.178 \\
\hline 2.5 & 3.250 & 3.850 & 3.666 & 0.184 \\
\hline 4.0 & 3.300 & 3.960 & 3.769 & 0.191 \\
\hline 5.0 & 3.400 & 4.180 & 3.977 & 0.203 \\
\hline 7.0 & 3.500 & 4.400 & 4.185 & 0.215 \\
\hline
\end{tabular}

The OIS zero rates are expressed with continuous compounding while all forward and forward spread rates are expressed with annual compounding. The OIS zero rates and LIBOR forward rates are exact. OIS zero rates and LIBOR forward rates for maturities other than those given are determined using linear interpolation. The rates in the final two columns are rounded values calculated from the given OIS zero rates and LIBOR forward rates

Table 2 Reversion rates, volatilities, and correlation for the examples

\begin{tabular}{l|l}
\hline OIS reversion rate, $a_{r}$ & 0.22 \\
\hline OIS volatility, $\sigma_{r}$ & 0.25 \\
\hline Spread reversion rate, $a_{s}$ & 0.10 \\
\hline Spread volatility, $\sigma_{s}$ & 0.20 \\
\hline $\begin{array}{l}\text { Correlation between OIS and } \\
\text { spread, } \rho\end{array}$ & 0.05 \\
\hline
\end{tabular}


of changes in time $\Delta t^{17}$

$$
\begin{aligned}
& p_{u}=\frac{1}{6}+\frac{1}{2}\left(a_{r}^{2} j^{2} \Delta t^{2}-a_{r} j \Delta t\right) \\
& p_{m}=\frac{2}{3}-a_{r}^{2} j^{2} \Delta t^{2} \\
& p_{d}=\frac{1}{6}+\frac{1}{2}\left(a_{r}^{2} j^{2} \Delta t^{2}+a_{r} j \Delta t\right)
\end{aligned}
$$

As soon as $j>0.184 /\left(a_{r} \Delta t\right)$, the branching process is changed so that $(i, j)$ leads to one of $(i+1, j),(i+1, j-1)$, and $(i+1, j-2)$. The transition probabilities to these three nodes are

$$
\begin{aligned}
& p_{u}=\frac{7}{6}+\frac{1}{2}\left(a_{r}^{2} j^{2} \Delta t^{2}-3 a_{r} j \Delta t\right) \\
& p_{m}=-\frac{1}{3}-a_{r}^{2} j^{2} \Delta t^{2}+2 a_{r} j \Delta t \\
& p_{d}=\frac{1}{6}+\frac{1}{2}\left(a_{r}^{2} j^{2} \Delta t^{2}-a_{r} j \Delta t\right)
\end{aligned}
$$

Similarly, as soon as $j<-0.184 /\left(a_{r} \Delta t\right)$ the branching process is changed so that $(i, j)$ leads to one of $(i+1, j+2),(i+1, j+1)$, and $(i+1, j)$. The transition probabilities to these three nodes are

$$
\begin{aligned}
& p_{u}=\frac{1}{6}+\frac{1}{2}\left(a_{r}^{2} j^{2} \Delta t^{2}+a_{r} j \Delta t\right) \\
& p_{m}=-\frac{1}{3}-a_{r}^{2} j^{2} \Delta t^{2}-2 a_{r} j \Delta t \\
& p_{d}=\frac{7}{6}+\frac{1}{2}\left(a_{r}^{2} j^{2} \Delta t^{2}+3 a_{r} j \Delta t\right)
\end{aligned}
$$

We then use an iterative procedure to calculate in succession the amount that the $x$-nodes at each time step must be shifted, $\alpha_{0}, \alpha_{\Delta t}, \alpha_{2 \Delta t}, \ldots$, so that the OIS term structure is matched. The first value, $\alpha_{0}$, is chosen so that the tree correctly prices a discount bond maturing $\Delta t$. The second value, $\alpha_{\Delta t}$, is chosen so that the tree correctly prices a discount bond maturing $2 \Delta t$, and so on.

Arrow-Debreu prices facilitate the calculation. The Arrow-Debreu price for a node is the price of a security that pays off $\$ 1$ if the node is reached and zero otherwise. Define $A_{i, j}$ as the Arrow-Debreu price for node $(i, j)$ and define $r_{i, j}$ as the $\Delta t$-maturity interest rate at node $(i, j)$. The value of $\alpha_{i \Delta t}$ can be calculated using an iterative search procedure from the $A_{i, j}$ and the price at time zero, $P_{i+1}$, of a bond maturing at time $(i+1) \Delta t$ using

\footnotetext{
${ }^{17}$ See for example Hull ([10], p. 725).
} 


$$
P_{i+1}=\sum_{j} A_{i, j} \exp \left(-r_{i, j} \Delta t\right)
$$

in conjunction with

$$
r_{i, j}=\exp \left(\alpha_{i \Delta t}+j \Delta x\right)
$$

where the summation in Eq. (3) is over all $j$ at time $i \Delta t$. The Arrow-Debreu prices can then be updated using

$$
A_{i+1, k}=\sum_{j} A_{i, j} p_{j, k} \exp \left(-r_{i, j} \Delta t\right)
$$

where $p(j, k)$ is the probability of branching from $(i, j)$ to $(i+1, k)$, and the summation is over all $j$ at time $i \Delta t$. The Arrow-Debreu price at the base of the tree, $A_{0,0}$, is one. From this $\alpha_{0}$ can be calculated using Eqs. (3) and (4). The $A_{1, k}$ can then be calculated using Eqs. (4) and (5). After that $\alpha_{\Delta t}$ can be calculated using Eqs. (3) and (4), and so on.

It is then necessary to calculate the value of the 12-month OIS rate at each node (step 2 in the previous section). As the tree has six-month time steps, a two-period roll back is required in the case of our simple example. It is necessary to build a four-step tree. The value at the $j$ th node at time $4 \Delta t(=2)$ of a discount bond that pays $\$ 1$ at time $5 \Delta t(=2.5)$ is $\exp \left(-r_{4, j} \Delta t\right)$.

Discounting these values back to time $3 \Delta t(=1.5)$ gives the price of a one-year discount bond at each node at $3 \Delta t$ from which the bond's yield can be determined. This is repeated for a bond that pays $\$ 1$ at time $4 \Delta t$ resulting in the one-year yields at time $2 \Delta t$, and so on. The tree constructed so far and the values calculated are shown in Fig. $2 .{ }^{18}$

The next stage (step 3 in the previous section) is to construct a tree for the spread assuming that the expected future spread is zero (the preliminary tree). As in the case of the OIS tree, $\Delta t=0.5$ and $\Delta y=\sigma_{s} \sqrt{3 \Delta t}=0.2449$. The branching process and probabilities are calculated as for the OIS tree (with $a_{r}$ replaced by $a_{s}$ ).

A three-dimensional tree is then created (step 4 in the previous section) by combining the spread tree and the OIS tree assuming zero correlation. We denote the node at time $i \Delta t$ where $x=j \Delta x$ and $y=k \Delta y$ by node $(i, j, k)$. Consider for example node $(2,-2,2)$. This corresponds to node $(2,-2)$ in the OIS tree, node $I$ in Fig. 2, and node $(2,2)$ in the spread tree. The probabilities for the OIS tree are $p_{u}=0.0809, p_{m}=0.0583, p_{d}=0.8609$ and the branching process is to nodes where $j=0, j=-1$, and $j=-2$. The probabilities for the spread tree are $p_{u}=0.1217, p_{m}=0.6567, p_{d}=0.2217$ and the branching process is to nodes where $k=1, k=2$, and $k=3$. Denote $p_{u u}$ as the probability of the highest move in the OIS tree being combined with the highest move in the spread tree; $p_{u m}$ as the probability of the highest move in the OIS tree being combined with the middle move in the spread tree; and so on. The probability, $p_{u u}$ of moving from node $(2,-2,2)$ to

\footnotetext{
${ }^{18}$ More details on the construction of the tree can be found in Hull [10].
} 


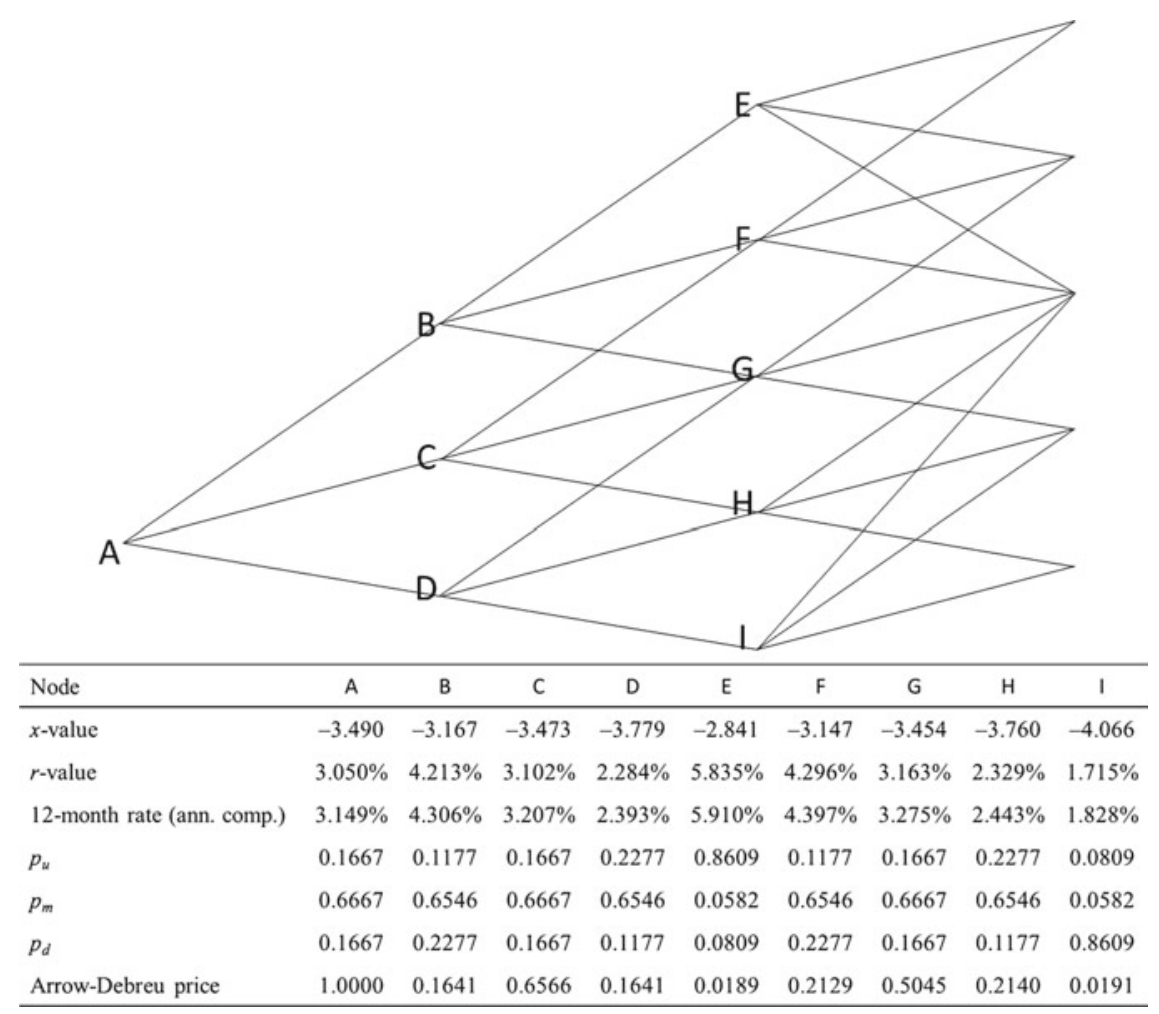

Fig. 2 Tree for OIS rates in three-step example

node $(3,0,3)$ is therefore $0.0809 \times 0.1217$ or 0.0098 ; the probability, $p_{\text {um }}$ of moving from node $(2,-2,2)$ to node $(3,0,2)$ is $0.0809 \times 0.6567$ or 0.0531 and so on. These (unadjusted) branching probabilities at node $(2,-2,2)$ are shown in Table $4 \mathrm{a}$.

The next stage (step 5 in the previous section) is to adjust the probabilities to build in correlation between the OIS rate and the spread (i.e., the correlation between $\mathrm{d} z_{r}$ and $\mathrm{d} z_{s}$ ). As explained in Hull and White [12], probabilities are changed as indicated in Table $3 .^{19}$ This leaves the marginal distributions unchanged. The resulting adjusted probabilities at node $(2,-2,2)$ are shown in Table $4 \mathrm{~b}$. In the example we are currently considering the adjusted probabilities are never negative. In practice negative probabilities do occur, but disappear as $\Delta t$ tends zero. They tend to occur only on the edges of the tree where the non-standard branching process is used and do not interfere with convergence. Our approach when negative probabilities are encountered at a node is to change the correlation at that node to the greatest (positive or negative) correlation that is consistent with non-negative probabilities.

\footnotetext{
${ }^{19}$ The procedure described in Hull and White [12] applies to trinomial trees. For binomial trees the analogous procedure is to increase $p_{u u}$ and $p_{d d}$ by $\varepsilon$ while decreasing $p_{u d}$ and $p_{d u}$ by $\varepsilon$ where $\varepsilon=\rho / 4$.
} 
Table 3 Adjustments to probabilities to reflect correlation in a three-dimensional trinomial tree

\begin{tabular}{l|l|l}
\hline Probability & Change when $\rho>0$ & Change when $\rho<0$ \\
\hline$p_{u u}$ & $+5 e$ & $+e$ \\
\hline$p_{u m}$ & $-4 e$ & $+4 e$ \\
\hline$p_{u d}$ & $-e$ & $-5 e$ \\
\hline$p_{m u}$ & $-4 e$ & $+4 e$ \\
\hline$p_{m m}$ & $+8 e$ & $-8 e$ \\
\hline$p_{m d}$ & $-4 e$ & $+4 e$ \\
\hline$p_{d u}$ & $-e$ & $-5 e$ \\
\hline$p_{d m}$ & $-4 e$ & $+4 e$ \\
\hline$p_{d d}$ & $+5 e$ & $+e$ \\
\hline
\end{tabular}

(e $e \rho / 36$ where $\rho$ is the correlation)

Table 4 (a) The unadjusted branching probabilities at node $(2,-2,2)$. The probabilities on the edge of the table are the branching probabilities at node $(2,-2)$ of the $r$-tree and $(2,2)$ of the $s$-tree. (b) The adjusted branching probabilities at node $(2,-2,2)$. The probabilities on the edge of the table are the branching probabilities at node $(2,-2)$ of the $r$-tree and $(2,2)$ of the $s$-tree. The adjustment is based on a correlation of 0.05 so $e=0.00139$

\begin{tabular}{|c|c|c|c|c|c|}
\hline \multicolumn{6}{|l|}{$a$} \\
\hline & & & \multicolumn{3}{|l|}{$r$-tree } \\
\hline & & & $p_{u}$ & $p_{m}$ & $p_{d}$ \\
\hline & & & 0.0809 & 0.0583 & 0.8609 \\
\hline \multirow[t]{3}{*}{$s$-tree } & $p_{u}$ & 0.1217 & 0.0098 & 0.0071 & 0.1047 \\
\hline & $p_{m}$ & 0.6567 & 0.0531 & 0.0383 & 0.5653 \\
\hline & $p_{d}$ & 0.2217 & 0.0179 & 0.0129 & 0.1908 \\
\hline \multicolumn{6}{|l|}{$b$} \\
\hline & & & \multicolumn{3}{|l|}{$r$-tree } \\
\hline & & & $p_{u}$ & $p_{m}$ & $p_{d}$ \\
\hline & & & 0.0809 & 0.0583 & 0.8609 \\
\hline \multirow[t]{3}{*}{$s$-tree } & $p_{u}$ & 0.1217 & 0.0168 & 0.0015 & 0.1033 \\
\hline & $p_{m}$ & 0.6567 & 0.0475 & 0.0494 & 0.5597 \\
\hline & $p_{d}$ & 0.2217 & 0.0165 & 0.0074 & 0.1978 \\
\hline
\end{tabular}

The tree constructed so far reflects actual OIS movements and artificial spread movements where the initial spread and expected future spread are zero. We are now in a position to calculate Arrow-Debreu prices for each node of the three-dimensional tree. These Arrow-Debreu prices remain the same when the positions of the spread nodes are changed because the Arrow-Debreu price for a node depends only on OIS rates and the probability of the node being reached. They are shown in Table 5.

The final stage involves shifting the position of the spread nodes so that the prices of all LIBOR FRAs with a fixed rate equal to the initial forward LIBOR rate are zero. An iterative procedure is used to calculate the adjustment to the values of $y$ 
Table 5 Arrow-Debreu prices for simple three-step example

\begin{tabular}{|c|c|c|c|c|c|c|c|}
\hline$i=1$ & $k=-1$ & $k=0$ & $k=1$ & & & & \\
\hline$j=1$ & 0.0260 & 0.1040 & 0.0342 & & & & \\
\hline$j=0$ & 0.1040 & 0.4487 & 0.1040 & & & & \\
\hline$j=-1$ & 0.0342 & 0.1040 & 0.0260 & & & & \\
\hline$i=2$ & $k=-2$ & $k=-1$ & $k=0$ & $k=1$ & $k=2$ & & \\
\hline$j=2$ & 0.0004 & 0.0037 & 0.0089 & 0.0051 & 0.0008 & & \\
\hline$j=1$ & 0.0045 & 0.0443 & 0.1064 & 0.0516 & 0.0061 & & \\
\hline$j=0$ & 0.0112 & 0.1100 & 0.2620 & 0.1100 & 0.0112 & & \\
\hline$j=-1$ & 0.0061 & 0.0518 & 0.1070 & 0.0445 & 0.0046 & & \\
\hline$j=-2$ & 0.0008 & 0.0052 & 0.0090 & 0.0037 & 0.0004 & & \\
\hline$i=3$ & $k=-3$ & $k=-2$ & $k=-1$ & $k=0$ & $k=1$ & $k=2$ & $k=3$ \\
\hline$j=2$ & 0.0001 & 0.0016 & 0.0085 & 0.0163 & 0.0109 & 0.0027 & 0.0002 \\
\hline$j=1$ & 0.0005 & 0.0094 & 0.0496 & 0.0932 & 0.0551 & 0.0116 & 0.0007 \\
\hline$j=0$ & 0.0012 & 0.0197 & 0.1016 & 0.1849 & 0.1016 & 0.0197 & 0.0012 \\
\hline$j=-1$ & 0.0008 & 0.0117 & 0.0557 & 0.0941 & 0.0501 & 0.0095 & 0.0005 \\
\hline$j=-2$ & 0.0002 & 0.0028 & 0.0111 & 0.0167 & 0.0087 & 0.0017 & 0.0001 \\
\hline
\end{tabular}

at each node at each time step, $\beta_{0}, \beta_{\Delta t}, \beta_{2 \Delta t}, \ldots$, so that the FRAs have a value of zero. Given that Arrow-Debreu prices have already been calculated this is a fairly straightforward search. When the $\alpha_{j \Delta t}$ are determined it is necessary to first consider $j=0$, then $j=1$, then $j=2$, and so on because the $\alpha$-value at a particular time depends on the $\alpha$-values at earlier times. The $\beta$-values however are independent of each other and can be determined in any order, or as needed. In the case of our example, $\beta_{0}=-6.493, \beta_{\Delta t}=-6.459, \beta_{2 \Delta t}=-6.426, \beta_{3 \Delta t}=-6.395$.

\section{Valuation of a Spread Option}

To illustrate convergence, we use the tree to calculate the value of a European call option that pays off 100 times $\max (s-0.002,0)$ at time $T$ where $s$ is the spread. First, we let $T=1.5$ years and use the three-step tree developed in the previous section. At the third step of the tree we calculate the spread at each node. The spread at node $(3, j, k)$ is $\exp [\phi(3 \Delta t)+k \Delta y]$. These values are shown in the second line of Table 6 . Once the spread values have been determined the option payoffs, 100 times $\max (s-0.002,0)$, at each node are calculated. These values are shown in the rest of Table 6. The option value is found by multiplying each option payoff by the corresponding Arrow-Debreu price in Table 5 and summing the values. The resulting option value is 0.00670 . Table 7 shows how, for a 1.5 - and 5 -year spread option, the value converges as the number of time steps per year is increased. 
Table 6 Spread and spread option payoff at time 1.5 years when spread option is evaluated using a three-step tree

\begin{tabular}{l|l|l|l|l|l|l|l}
\hline$i=3$ & $k=-3$ & $k=-2$ & $k=-1$ & $k=0$ & $k=1$ & $k=2$ & $k=3$ \\
\hline Spread & 0.0008 & 0.0010 & 0.0013 & 0.0017 & 0.0021 & 0.0027 & 0.0035 \\
\hline$j=2$ & 0.0000 & 0.0000 & 0.0000 & 0.0000 & 0.0133 & 0.0725 & 0.1482 \\
\hline$j=1$ & 0.0000 & 0.0000 & 0.0000 & 0.0000 & 0.0133 & 0.0725 & 0.1482 \\
\hline$j=0$ & 0.0000 & 0.0000 & 0.0000 & 0.0000 & 0.0133 & 0.0725 & 0.1482 \\
\hline$j=-1$ & 0.0000 & 0.0000 & 0.0000 & 0.0000 & 0.0133 & 0.0725 & 0.1482 \\
\hline$j=-2$ & 0.0000 & 0.0000 & 0.0000 & 0.0000 & 0.0133 & 0.0725 & 0.1482 \\
\hline
\end{tabular}

Table 7 Value of a European spread option paying off 100 times the greater of the spread less 0.002 and zero

\begin{tabular}{l|l|l}
\hline Time steps per year & 1.5 -year option & 5-year option \\
\hline 2 & 0.00670 & 0.0310 \\
\hline 4 & 0.00564 & 0.0312 \\
\hline 8 & 0.00621 & 0.0313 \\
\hline 16 & 0.00592 & 0.0313 \\
\hline 32 & 0.00596 & 0.0313 \\
\hline
\end{tabular}

The market data used to build the tree is given in Tables 1 and 2

Table 8 Value of a five-year European spread option paying off 100 times the greater of the spread less 0.002 and zero

\begin{tabular}{l|l|l|l|l|l|l|l}
\hline $\begin{array}{l}\text { Spread } \\
\text { volatility }\end{array}$ & \multicolumn{6}{l}{ Spread/OIS correlation } \\
\hline & -0.75 & -0.50 & -0.25 & 0 & 0.25 & 0.5 & 0.75 \\
\hline 0.05 & 0.0141 & 0.0142 & 0.0142 & 0.0143 & 0.0143 & 0.0144 & 0.0144 \\
\hline 0.10 & 0.0193 & 0.0194 & 0.0195 & 0.0195 & 0.0196 & 0.0196 & 0.0197 \\
\hline 0.15 & 0.0250 & 0.0252 & 0.0253 & 0.0254 & 0.0254 & 0.0255 & 0.0256 \\
\hline 0.20 & 0.0308 & 0.0309 & 0.0311 & 0.0313 & 0.0314 & 0.0316 & 0.0317 \\
\hline 0.25 & 0.0367 & 0.0369 & 0.0371 & 0.0373 & 0.0374 & 0.0376 & 0.0377 \\
\hline
\end{tabular}

The market data used to build the tree are given in Tables 1 and 2 except that the volatility of the spread and the correlation between the spread and the OIS rate are as given in this table. The number of time steps is 32 per year

Table 8 shows how the spread option price is affected by the assumed correlation and the volatility of the spread. All of the input parameters are as given in Tables 1 and 2 except that correlations between -0.75 and 0.75 , and spread volatilities between 0.05 and 0.25 are considered. As might be expected the spread option price is very sensitive to the spread volatility. However, it is not very sensitive to the correlation. The reason for this is that changing the correlation primarily affects the Arrow-Debreu prices and leaves the option payoffs almost unchanged. Increasing the correlation increases the Arrow-Debreu prices on one diagonal of the final nodes and decreases them on the other diagonal. For example, in the three-step tree used 
to evaluate the option, the Arrow-Debreu price for nodes $(3,2,3)$ and $(3,-2,-3)$ increase while those for nodes $(3,-2,3)$ and $(3,2,-3)$ decrease. Since the option payoffs at nodes $(3,2,3)$ and $(3,-2,3)$ are the same, the changes on the ArrowDebreu prices offset one another resulting in only a small correlation effect.

\section{Bermudan Swap Option}

We now consider how the valuation of a Bermudan swap option is affected by a stochastic spread in a low-interest-rate environment such as that experienced in the years following 2009. Bermudan swap options are popular instruments where the holder has the right to enter into a particular swap on a number of different swap payment dates.

The valuation procedure involves rolling back through the tree calculating both the swap price and (where appropriate) the option price. The swap's value is set equal to zero at the nodes on the swap's maturity date. The value at earlier nodes is calculated by rolling back adding in the present value of the next payment on each reset date. The option's value is set equal to $\max (S, 0)$ where $S$ is the swap value at the option's maturity. It is then set equal to $\max (S, V)$ for nodes on exercise dates where $S$ is the swap value and $V$ is the value of the option given by the roll back procedure.

We assume an OIS term structure that increases linearly from 15 basis points at time zero to 250 basis points at time 10 years. The OIS zero rate for maturity $t$ is therefore

$$
0.0015+\frac{0.0235 t}{10}
$$

The process followed by the instantaneous OIS rate was similar to that derived by Deguillaume, Rebonato and Pogodin [7], and Hull and White [16]. For short rates between 0 and $1.5 \%$, changes in the rate are assumed to be lognormal with a volatility of $100 \%$. Between $1.5 \%$ and $6 \%$ changes in the short rate are assumed to be normal with the standard deviation of rate moves in time $\Delta t$ being $0.015 \sqrt{\Delta t}$. Above $6 \%$ rate moves were assumed to be lognormal with volatility $25 \%$. This pattern of the short rate's variability is shown in Fig. 3.

The spread between the forward 12-month OIS and the forward 12-month LIBOR was assumed to be 50 basis points for all maturities. The process assumed for the 12-month LIBOR-OIS spread, $s$, is that used in the example in Sects. 4 and 5

$$
\mathrm{d} \ln (s)=a_{s}[\phi(t)-\ln (s)]+\sigma_{s} \mathrm{~d} z_{s}
$$




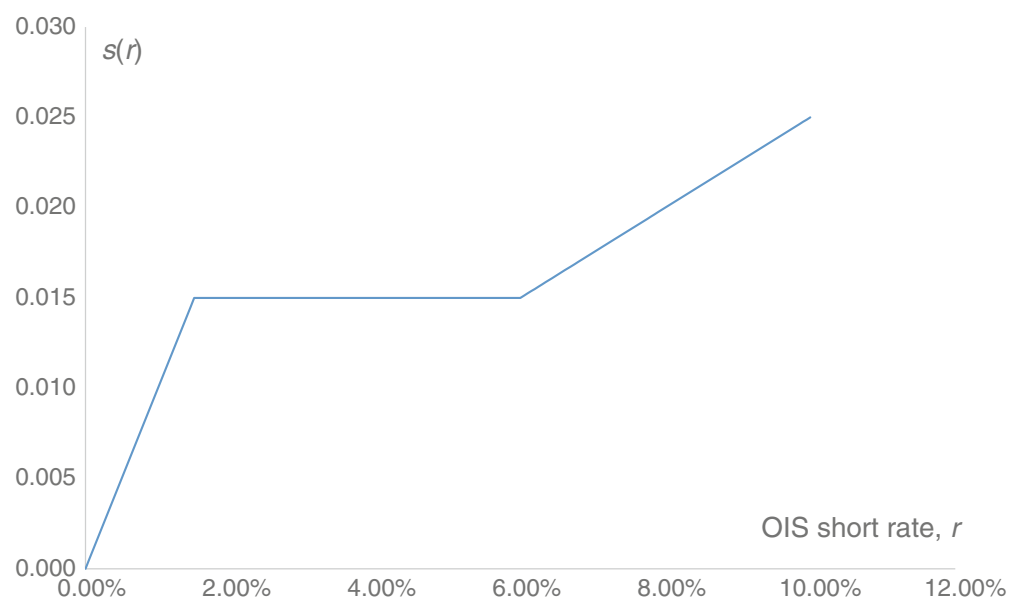

Fig. 3 Variability assumed for short OIS rate, $r$, in Bermudan swap option valuation. The standard deviation of the short rate in time $\Delta t$ is $s(r) \sqrt{\Delta t}$

Table 9 (a) Value in a low-interest rate environment, of a receive-fixed Bermudan swap option on a 5-year annual-pay swap where the notional principal is 100 and the option can be exercised at times 1,2, and 3 years. The swap rate is $1.5 \%$. (b) Value in a low-interest-rate environment of a received-fixed Bermudan swap option on a 10-year annual-pay swap where the notional principal is 100 and the option can be exercised at times 1, 2, 3, 4, and 5 years. The swap rate is $3.0 \%$

\begin{tabular}{|c|c|c|c|c|c|c|c|}
\hline Spread & \multicolumn{7}{|c|}{ Spread/OIS correlation } \\
\hline \multicolumn{8}{|l|}{$a$} \\
\hline & -0.5 & -0.25 & -0.1 & 0 & 0.1 & 0.25 & 0.5 \\
\hline 0 & 0.398 & 0.398 & 0.398 & 0.398 & 0.398 & 0.398 & 0.398 \\
\hline 0.3 & 0.333 & 0.371 & 0.393 & 0.407 & 0.421 & 0.441 & 0.473 \\
\hline 0.5 & 0.310 & 0.373 & 0.407 & 0.429 & 0.449 & 0.480 & 0.527 \\
\hline 0.7 & 0.309 & 0.389 & 0.432 & 0.459 & 0.485 & 0.522 & 0.580 \\
\hline \multicolumn{8}{|l|}{$b$} \\
\hline & -0.5 & -0.25 & -0.1 & 0 & 0.1 & 0.25 & 0.5 \\
\hline 0 & 2.217 & 2.218 & 2.218 & 2.218 & 2.218 & 2.218 & 2.218 \\
\hline 0.3 & 2.100 & 2.164 & 2.201 & 2.225 & 2.248 & 2.283 & 2.339 \\
\hline 0.5 & 2.031 & 2.141 & 2.203 & 2.242 & 2.280 & 2.335 & 2.421 \\
\hline 0.7 & 1.980 & 2.134 & 2.218 & 2.271 & 2.321 & 2.392 & 2.503 \\
\hline
\end{tabular}

A maximum likelihood analysis of data on the 12-month LIBOR-OIS spread over the 2012 to 2014 period indicates that the behavior of the spread can be approximately described by a high volatility in conjunction with a high reversion rate. We set $a_{s}$ equal to 0.4 and considered values of $\sigma_{s}$ equal to $0.30,0.50$, and 0.70 . A number of alternative correlations between the spread process and the OIS process were also 
considered. We find that correlation of about -0.1 between one month OIS and the 12-month LIBOR OIS spread is indicated by the data. ${ }^{20}$

We consider two cases:

1. A $3 \times 5$ swap option. The underlying swap lasts 5 years and involves 12 -month LIBOR being paid and a fixed rate of $1.5 \%$ being received. The option to enter into the swap can be exercised at the end of years 1,2 , and 3 .

2. A $5 \times 10$ swap option. The underlying swap lasts 10 years and involves 12 -month LIBOR being paid and a fixed rate of $3.0 \%$ being received. The option to enter into the swap can be exercised at the end of years $1,2,3,4$, and 5.

Table 9a shows results for the $3 \times 5$ swap option. In this case, even when the correlation between the spread rate and the OIS rate is relatively small, a stochastic spread is liable to change the price by $5-10 \%$. Table 9 b shows results for the $5 \times 10$ swap option. In this case, the percentage impact of a stochastic spread is smaller. This is because the spread, as a proportion of the average of the relevant forward OIS rates, is lower. The results in both tables are based on 32 time steps per year. As the level of OIS rates increases the impact of a stochastic spread becomes smaller in both Table $9 a, b$.

Comparing Tables 8 and 9, we see that the correlation between the OIS rate and the spread has a much bigger effect on the valuation of a Bermudan swap option than on the valuation of a spread option. For a spread option we argued that option payoffs for high Arrow-Debreu prices tend to offset those for low Arrow-Debreu prices. This is not the case for a Bermudan swap option because the payoff depends on the LIBOR rate, which depends on the OIS rate as well as the spread.

\section{Conclusions}

For investment grade companies it is well known that the hazard rate is an increasing function of time. This means that the credit spread applicable to borrowing by AArated banks from other banks is an increasing function of maturity. Since 2008, markets have recognized this with the result that the LIBOR-OIS spread has been an increasing function of tenor.

Since 2008, practitioners have also switched from LIBOR discounting to OIS discounting. This means that two zero curves have to be modelled when most interest rate derivatives are valued. Many practitioners assume that the relevant LIBOR-OIS spread is either constant or deterministic. Our research shows that this is liable to lead to inaccurate pricing, particularly in the current low interest rate environment.

The tree approach we have presented provides an alternative to Monte Carlo simulation for simultaneously modelling spreads and OIS rates. It can be regarded as

\footnotetext{
${ }^{20}$ Because of the way LIBOR is calculated, daily LIBOR changes can be less volatile than the corresponding daily OIS changes (particularly if the Fed is not targeting a particular overnight rate). In some circumstances, it may be appropriate to consider changes over periods longer than one day when estimating the correlation.
} 
an extension of the explicit finite difference method and is particularly useful when American-style derivatives are valued. It avoids the need to use techniques such as those suggested by Longstaff and Schwartz [19] and Andersen (2000) for handling early exercise within a Monte Carlo simulation.

Implying all the model parameters from market data is not likely to be feasible. One reasonable approach is to use historical data to determine the spread process and its correlation with the OIS process so that only the parameters driving the OIS process are implied from the market. The model can then be used in the same way that two-dimensional tree models for LIBOR were used pre-crisis.

Acknowledgements We are grateful to the Global Risk Institute in Financial Services for funding this research.

The KPMG Center of Excellence in Risk Management is acknowledged for organizing the conference "Challenges in Derivatives Markets - Fixed Income Modeling, Valuation Adjustments, Risk Management, and Regulation".

Open Access This chapter is distributed under the terms of the Creative Commons Attribution 4.0 International License (http://creativecommons.org/licenses/by/4.0/), which permits use, duplication, adaptation, distribution and reproduction in any medium or format, as long as you give appropriate credit to the original author(s) and the source, a link is provided to the Creative Commons license and any changes made are indicated.

The images or other third party material in this chapter are included in the work's Creative Commons license, unless indicated otherwise in the credit line; if such material is not included in the work's Creative Commons license and the respective action is not permitted by statutory regulation, users will need to obtain permission from the license holder to duplicate, adapt or reproduce the material.

\section{References}

1. Ames, W.F.: Numerical Methods for Partial Differential Equations. Academic Press, New York (1977)

2. Anderson, L.: A simple approach to pricing Bermudan swap options in the multifactor LIBOR market model. J. Comput. Financ. 3(2), 1-32 (2000)

3. Black, F., Derman, E., Toy, W.: A one-factor model of interest rates and its application to treasury bond prices. Financ. Anal. J. 46(1), 1-32 (1990)

4. Black, F., Karasinski, P.: Bond and option pricing when short rates are lognormal. Financ. Anal. J. 47(4), 52-59 (1991)

5. Brigo, D., Mercurio, F.: Interest Rate Models: Theory and Practice: With Smile Inflation and Credit, 2nd edn. Springer, Berlin (2007)

6. Collin-Dufresne, P., Solnik, B.: On the term structure of default premia in the swap and LIBOR market. J. Financ. 56(3), 1095-1115 (2001)

7. DeGuillaume, N., Rebonato, R., Pogudin, A.: The nature of the dependence of the magnitude of rate moves on the level of rates: a universal relationship. Quant. Financ. 13(3), 351-367 (2013)

8. Hainaut, D., MacGilchrist, R.: An interest rate tree driven by a Lévy process. J. Deriv. 18(2), 33-45 (2010)

9. Ho, T.S.Y., Lee, S.-B.: Term structure movements and pricing interest rate contingent claims. J. Financ. 41, 1011-1029 (1986)

10. Hull, J.: Options, Futures and Other Derivatives, 9th edn. Pearson, New York (2015) 
11. Hull, J., White, A.: Numerical procedures for implementing term structure models I. J. Deriv. 2(1), 7-16 (1994)

12. Hull, J., White, A.: Numerical procedures for implementing term structure models II. J. Deriv. 2(2), 37-48 (1994)

13. Hull, J., White, A.: Using Hull-White interest rate trees. J. Deriv. 3(3), 26-36 (1996)

14. Hull, J., White, A.: The general Hull-White model and super calibration. Financ. Anal. J. 57(6), 34-43 (2001)

15. Hull, J., White, A.: LIBOR vs. OIS: The derivatives discounting dilemma. J. Invest. Manag. 11(3), 14-27 (2013)

16. Hull, J., White, A.: A generalized procedure for building trees for the short rate and its application to determining market implied volatility functions. Quant. Financ. 15(3), 443-454 (2015)

17. Hull, J., White, A.: OIS discounting, interest rate derivatives, and the modeling of stochastic interest rate spreads. J. Invest. Manag. 13(1), 13-20 (2015)

18. Kalotay, A.J., Williams, G.O., Fabozzi, F.J.: A model for valuing bonds and embedded options. Financ. Anal. J. 49(3), 35-46 (1993)

19. Longstaff, F.A., Schwartz, E.S.: Valuing American options by Monte Carlo simulation: a simple least squares approach. Rev. Financ. Stud. 14(1), 113-147 (2001) 